\title{
Miniaturized Human Insertable Cardiac Monitoring System with Wireless Power Transmission Technique
}

\author{
Jong-Ha Lee \\ Department of Biomedical Engineering, School of Medicine, Keimyung University, Daegu 704-701, Republic of Korea
}

Correspondence should be addressed to Jong-Ha Lee; segeberg@gmail.com

Received 28 January 2015; Revised 26 April 2015; Accepted 12 May 2015

Academic Editor: Toshiyo Tamura

Copyright (c) 2016 Jong-Ha Lee. This is an open access article distributed under the Creative Commons Attribution License, which permits unrestricted use, distribution, and reproduction in any medium, provided the original work is properly cited.

\begin{abstract}
Prolonged monitoring is more likely to diagnose atrial fibrillation accurately than intermittent or short-term monitoring. In this study, an implantable electrocardiograph (ECG) sensor to monitor atrial fibrillation patients in real time was developed. The implantable sensor is composed of a micro controller unit, an analog-to-digital converter, a signal transmitter, an antenna, and two electrodes. The sensor detects ECG signals from the two electrodes and transmits these to an external receiver carried by the patient. Because the sensor continuously transmits signals, its battery consumption rate is extremely high; therefore, the sensor includes a wireless power transmission module that allows it to charge wirelessly from an external power source. The integrated sensor has the approximate dimensions $3 \mathrm{~mm} \times 4 \mathrm{~mm} \times 14 \mathrm{~mm}$, which is small enough to be inserted into a patient without the need for major surgery. The signal and power transmission data sampling rate and frequency of the unit are 300 samples/s and $430 \mathrm{~Hz}$, respectively. To validate the developed sensor, experiments were conducted on small animals.
\end{abstract}

\section{Introduction}

There are numerous medical problems whose treatment requires the constant monitoring of vital signs from several body organs. Although patients are typically hospitalized and kept under observation using wired equipment to measure vital signs, remote patients must stay at home along with expensive monitoring equipment and dedicated medical staff, which increases medical expenditure and reduces human resources available at the hospital. Several studies have therefore been conducted recently in researching and developing wearable and implantable biomedical devices, and progress in this field has provided benefits in terms of lower costs, freer patient movement, and uninterrupted diagnostic data streams for medical monitoring. Wireless biomedical devices can provide enhanced mobility and efficiency with minimum disruption of monitored data [1], and networks based on biomedical sensors can create effective solutions for distributing patient information along multiple platforms.

Diabetes and cardiovascular diseases are two health conditions that require effective, round-the-clock monitoring. Twenty-four percent of the population of developed countries has diabetes and related complications such as cardiovascular diseases, making this a widespread health issue that can only be addressed through active monitoring of blood glucose levels (BGL) [2].

The vital signs that are most often monitored in health diagnostics are:

(i) blood glucose level;

(ii) blood pressure and pulse rate;

(iii) electrocardiograph (ECG);

(iv) respiration efficacy.

Advancements in the use of wireless technologies in biomedical implant design have opened avenues for marked improvement in medical care and diagnostic systems as wired equipment is replaced with implanted on-body sensors. Biomedical implant-based monitoring systems can wirelessly transmit data consisting of critical information related to patient health. Implant-based vital-sign monitoring allows for round-the-clock monitoring and health management, with updates provided on handheld devices using wireless protocols. A range of medical diagnoses can be performed using implants through the monitoring of parameters such 
as blood pressure, glucose level, and cardiac response. However, efficient invasive monitoring using wireless biomedical implants comes with numerous challenges that must be addressed beforehand.

Recently there are several noninvasive ECG monitoring in the market such as ZioPatch. The patch fuses powerefficient electronics and standardized communication. The ECG patch records up to 3 lead ECG signals and tissuecontact impedance and includes a 3D accelerometer for physical activity monitoring. The data are processed and analyzed locally, and relevant events and information are transmitted through BLE wireless link. However, the patchtype noninvasive sensor is not suitable for users to hold 24 hours. The advantage of implantable sensor is more suitable to continuously monitor the human for 24 hours 7 days. Research on implants has progressed significantly in the last decade and is being actively pursued owing to the viability of implants in a broad range of applications including medicine, health, and sports. On-time diagnostics have become a major benefit for patients with chronic diseases as the continuous monitoring of health indicators can significantly assist in curtailing emergency events. The design of wireless biomedical implants is a difficult task, however, as there are many challenges that must be addressed for operational systems. Some important key issues are as follows.

(i) Power Requirements. Biomedical implants vary in power requirements based on their operational issues. To improve implant lifetime and range of communication, and because excessive power dissipation by a medical implant can seriously increase the chances of tissue damage [3], low power consumption is generally sought. Implants can be powered using batteries or wireless power transfer; however, batteries are bulky and hazardous and require recurrent replacement, while wireless power allows for continuous power transfer, making it more suitable for 24-hour monitoring systems.

(ii) Sensors and Communication. Accurately reading and monitoring signals from a human body require sensitive transducers and amplification units. Algorithms for the interpretation of signals must also be carefully designed to cater to any signal pattern anomalies in a timely manner. Wireless system must also be carefully designed to comply with power requirements and transmission ranges [4].

(iii) Implant Size. Implant size has serious impacts on overall design [5]. Power requirements, carrier frequency, and transducer design are all primarily governed by the size of the implant, which in turn is governed by where in the body the implant is placed. Smaller implants also allow for minimally invasive surgical procedures.

(iv) Reliability. The reliability and efficacy of an implantable medical device are paramount for enabling active monitoring and timely warning under emergency situations. In cardiac cases in particular, emergencies can be avoided through the use of implants that can produce reliable measurements. Good reliability will also reduce the need for periodic surgery in order to install replacements. To sustain future requirements ensuring the viability of biomedical implant technology, prolonged reliability is essential.

The above concerns must be kept in mind when designing an implant as they represent the major limiting factors for advances in implant technology. In this study, an implantable ECG sensor using wireless communication and power transmission was developed. With the increased pace of living in contemporary society and the related reliance on tobacco, alcohol, and caffeine, the number of patients with heart conditions such as arrhythmia is increasing. Arrhythmia (also known as cardiac dysrhythmia) is caused by an abnormal ejection fraction and presents as an irregular heartbeat that is either faster (tachycardia) or slower (bradycardia) than the usual heart rate. It can occur unexpectedly anytime and anywhere and can lead to shortness of breath, dizziness, and fainting; in serious cases, it can cause sudden cardiac arrest owing to noncontraction of the ventricles, resulting in a life-threatening myocardial infarction (heart attack). An electrocardiography (or electrocardiograph) (ECG) can be used to detect cardiac abnormalities and thus predict arrhythmia. The ECG produces a graph on which changes in electrical potential associated with the pattern of the heartbeat are recorded. Measurements using an ECG can be performed with either patch- or insertion-type ECG sensors. The most widely used ECG sensor is the standard patchtype 12-lead ECG, in which electrodes are attached to the four limbs and to the anterior chest near the heart in order to measure and record ECG signals using standard limb, unipolar limb, and chest leads. However, in the case of arrhythmia a short-term ECG measurement is of little help because of the short duration of symptoms; it is therefore necessary to use an ECG device that can be carried by the patient and has electrodes attached to the body's surface. The heart rhythm can be recorded using either a Holter monitor or an implantable loop recorder (ILR) surgically inserted under the skin. Unfortunately, the Holter monitor is inconvenient as it disrupts daily activities because it must be worn constantly. Although the insertion-type ILR is more comfortable as it is implanted into the body and does not need to be carried, its use requires surgical intervention for implantation, which raises safety and confidence issues. In addition, a similar surgical procedure is necessary at the end of battery life to either replace or remove it, which again raises safety and cost issues.

The drawbacks of ILR can be overcome through the use of a quasipermanent battery that is recharged via wireless power transmission. While this is technically possible, further investigation of effects on the human body must be conducted before such systems can be considered safe and reliable. Therefore, in this study an insertion-type wireless ECG sensor was developed and its performance within a human body phantom was tested using a thermal imaging camera. Further tests of the implanted sensor were then conducted on an animal model. Based on the results of these tests, it was 
possible to identify any potential problems that could occur in the use of a wireless ECG sensor.

\section{System Design Concepts}

In this section, the design concept of the implantable ECG sensor is presented. Numerical simulations were performed to verify the principle behind sensor and tactile images of phantom tissue inclusions were obtained.

2.1. Cardiac ECG Measurement and Electrodes. Cardiac activity is normally monitored by recording electrocardiograph (ECG) signals in a clinical setting, which requires the physical presence of the patient at the facility. The ECG signal is composed of multiple electrical activities that begin from the sinus at the top of the right atrium. The signal is generated from the sinus node and propagates through the atrioventricular (AV) node. One cardiac cycle consists of a $\mathrm{P}$ wave, a $\mathrm{T}$ wave, and a QRS complex, all of which were identified by Willem Einthoven. When a sinus node releases an electrical impulse, it creates the basis for an atrial depolarization leading to atrial contraction, which is sensed as a $\mathrm{P}$ wave. The signal then passes through the $\mathrm{AV}$ node, where the QRS complex signal is induced by ventricular depolarization and is followed by generation of a $\mathrm{T}$ wave from the repolarization of the ventricular node.

Monitoring heart activity through ECG signals is carried out using at least three electrodes placed on specific points on the skin in order to sense electrical signals generated by heart constituents. The Holter monitor is one such diagnostic device and is commonly employed for active monitoring of heart activity after major heart procedures [6]. Holter monitors have proven technologically capable but are large, must be connected to electrodes using wires that limit free movement of the patient, and require continuous placement of electrodes for long-term monitoring. Standard Holter monitors are therefore incapable of providing smooth and seamless unobtrusive continuous monitoring, and although Holter devices have evolved over the past few years into complete wire-free miniaturized modules, they still require further improvements to ensure totally unobtrusive monitoring architecture.

In this study, $\mathrm{Ag} / \mathrm{AgCl}$ electrodes were used. An ECG traces the electrical potential differences between electrodes placed on the body's surface; however, the action potential that gives rise to the contraction and relaxation of the cardiac muscle is only about $1 \mathrm{mV}$, which is extremely difficult to measure. It is therefore necessary to amplify electrocardiographic data to make it easily perceivable to the human eye; this is done through the use of an operational amplifier (opamp), which amplifies an input electrical potential in order to produce an output potential augmented to the level desired by the user. In this study, an instrumentation amplifier using op-amps was fabricated and configured to amplify the microfine ECG by a factor of about 100 using a band-pass filter (BPS). The current consumption of the proposed ECG sensor is about $11 \mathrm{~mA}$ and its noise generation is inversely proportional to the length of the wireless communication antenna inside the sensor. Traditionally, the ECG sensor needs three electrodes as positive, negative, and ground. In the proposed system, however, the ground electrode is emitted and only two electrodes were used. On the main sensor body, in the left and right sides of the body, two electrodes were attached.

2.2. Telemetry Methods. Currently used systems for health monitoring employ a variety of methods to relay information between the sensors and the data display module. Data are normally shared between these two units using wires, which increases the redundancy of the system and limits movement of the patient. Although wire-based equipment provides a robust means for communication in health monitoring systems and is low-cost, it reduces the ability for normal movement of a patient in her everyday routines.

Another problem arising in wired systems is the improper connection of wires for various reasons, which can seriously interrupt the system and pose serious consequences for the patient. Continuous improvisation and research are being carried out to develop smart health monitoring systems and many alternative communication techniques have emerged, with wireless communication being the most suitable communication method for curtailing the need for wired connections between sensors and equipment. Wireless technologies enable intrabody communication to complement systems for continual health monitoring without the need for admitting patients and attaching wires. Wireless communication allows for real-time monitoring of vital signs on an unwired display device in proximity to the patient as well as for remote observation by a doctor via the internet. Wireless connectivity can also help patients to track their own health indicators using smart-phones or PDAs connected to implants or wearable sensors in real-time. This can result in better health management and prompt alerts in the case of health related emergencies.

Biomedical implanting is a vibrant technology that has shown promise in improving real-time medical diagnostics. Research has shown that implants can be used to provide a feedback control; for example, an implant variant was used to record neural signals in brain-machine interfaces in order to control prostheses or paralyzed limbs [6, 7]. Implants that use wireless communication have been shown to significantly reduce drawbacks attributed to wire connections, as has been reported with wired deep brain systems, implantable cardiodefibrillators, and pacemakers [8].

In this study, the Medical Implant Communication Service (MICS) was used as a communications protocol. MICS operates in the frequency range of $402-405 \mathrm{MHz}$ and is normally used for communication between body-worn monitoring systems and implants. Implantable antennas in this frequency range have been developed to transmit data from pacemakers and cardiac sensors; however, regulatory restrictions in hospitals have limited their full utilization in wireless body area networks (WBANs).

2.3. Wireless Power for Biomedical Implants. Supplying adequate power to biomedical implants is currently the main 
challenge limiting functionality and performance in such devices. Power consumption affects many characteristics of an implant, including size, processing power, transmission range, and life span.

Batteries can power implants for long periods of time by exploiting design techniques that require extremely low power consumption. The average power consumption for a battery used in a pacemaker is about $8 \mu \mathrm{W}$ and the typical battery comprises $90 \%$ of the total size of the implant and requires periodic replacement through costly invasive surgery every few years $[9,10]$. Power-hungry implants such as mechanical pump-based cardiac and orthopedic implants require significant amounts of power to function, making batteries an ineffective power source option for such implants $[11,12]$. Thus, the prospects for and applicability of biomedical implant technology are currently severely limited by the unavailability of adequate power sources, a problem that can be successfully addressed by using wireless power transfer techniques capable of delivering uninterrupted power to ensure continuous monitoring and communication by implants.

In this study, a near-field wireless power transmission system was used. It is assumed that the radiated fields produced by inductive coupling are not rapidly changing; as the displacement current at low frequencies does not affect the generated fields, it can be ignored. This is generally called the quasistatic approximation. Using this approximation, the magnetic field was found to be concentrated in the vicinity of the source. Problems such as these can be analytically solved using the Biot-Savart law or by finding a solution using the diffusion equation.

Many techniques for implementing coupled power links have been reported in the literature. In one study [13], the authors used coupled self-resonant coils to power a $60 \mathrm{~W}$ bulb over a distance of $2 \mathrm{~m}$ with an efficiency of $40 \%$. This was accomplished by nonradiating magnetic induction using resonant loops: employing two identical helical coils as coupling elements, a standard Colpitts oscillator with a single copper wire loop inductive element was used to generate frequencies in the $\mathrm{MHz}$ range. The copper loop coupled inductive power to the source coil for further transmission and a light-bulb served as the load of the power transfer system. Experimental results showed that power transfer using nonradiative magnetic coupling could be achieved over a range of 8 9 times the radius of the coils. The authors also presented a quantitative model with an accuracy of around $5 \%$ for explaining the power transfer.

2.4. Circuit Design. An ECG traces the electrical potential differences between electrodes placed on the surface of a body. However, the action potential that gives rise to the contraction and relaxation of the cardiac muscle is about $1 \mathrm{mV}$ and thus extremely difficult to measure; in this design, operational amplifiers (op-amps) are used to amplify the electrocardiographic data. An op-amp amplifies an input electrical potential to the level desired by the user and produces an output potential augmented to this intended level. For this study an instrumentation amplifier was fabricated

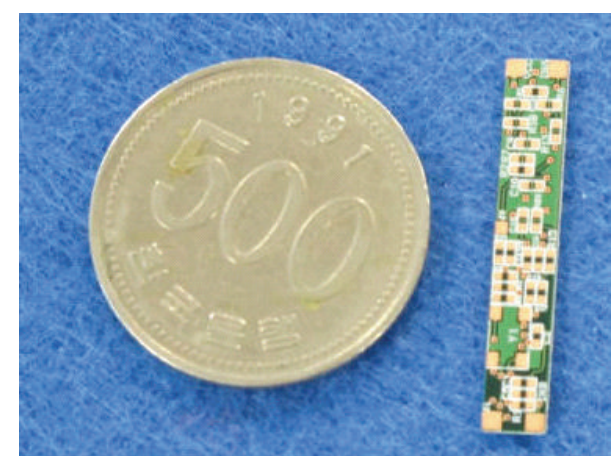

Figure 1: Circuit design of implantable ECG sensor.

using op-amps and configured to amplify microfine ECG signals by a factor of about 100 using a band-pass filter (BPF). The proposed ECG sensor has a current consumption of about $11 \mathrm{~mA}$ and a noise generation inversely proportional to the length of the wireless communication antenna inside the sensor. Figure 1 shows the circuit design of the implantable ECG sensor.

2.5. Packaging. The packaging materials selected must be biocompatible to avoid causing inflammation or necrosis of human tissues. Additionally, they need to satisfy the strength standards of the insertion location and should not absorb electric waves passing through them. It is also necessary to take packaging design into account in order to avoid any risk of damage to tissues caused by sensor insertion and postinsertion movements. Foreign-body sensation needs to be minimized by reducing the size of the object. As feedthrough needs to meet several requirements, conductivity should be ensured between the internal circuit and electrodes, noise should be minimized, and air-tightness should be maintained. In this study, the elasticity of polymer films was exploited to develop electrode sealing methods.

ECG sensor electrodes must have an electrode-toelectrode distance of $\geq 40 \mathrm{~mm}$ and an electrode width of $\geq 5 \mathrm{~mm}$. In order to satisfy the requirements for electrodes, they were fabricated using titanium. The rigid packaging materials need to be coated to protect tissues and must be hermetically joined using a proper joining technique to completely block any interaction between the interior of the human body and the sensor environment; to accomplish this, either adhesives or a laser can be used. In this study, adhesives were used to produce a packaging prototype. Polydimethylsiloxane (PDMS) and medical epoxy are suitable adhesives, while PDMS, parylene, polyethylene, glycol, and silicone can be used as coating materials as their biocompatibilities have been verified in numerous studies. Figure 2 shows the packaged implantable ECG sensor.

The full diagram of the proposed system is shown in Figure 3.

\section{Experimental Results}

3.1. Self-Sealing Air-Tightness Testing. Testing for self-sealing air-tightness was performed in two steps. In the first step, 


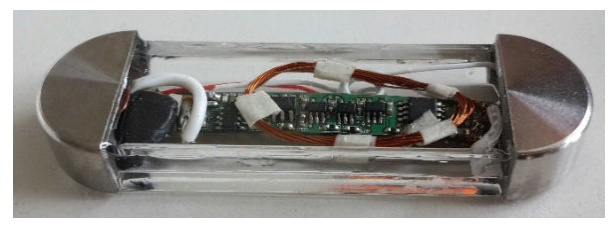

FIgURe 2: Packaged implantable ECG sensor.

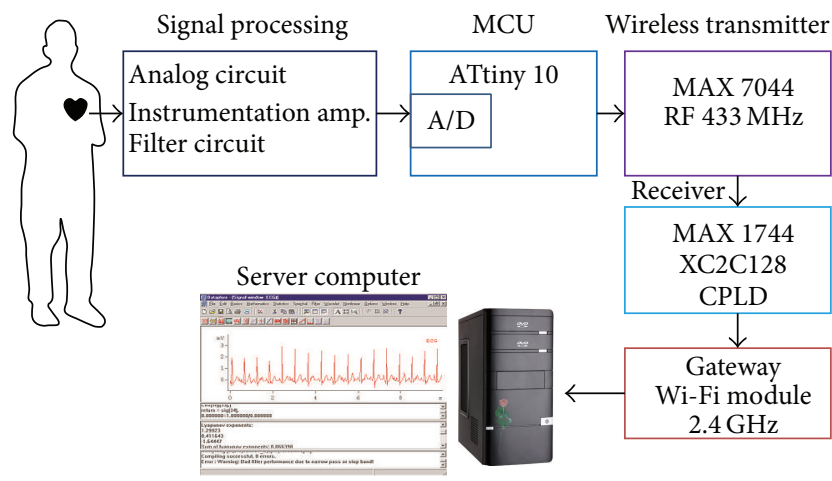

FigURE 3: Implantable sensor and workstation diagram.

the packaging was submersed in deionized (DI) water for one hour with no sensor included, and in the second test the packaging containing the sensor was submersed in DI water for five hours. Results of tests show that the materials joined with adhesives are air-tight. Figure 3 shows the self-sealing test using the packaged sensor.

3.2. Thermal Testing. Coil charging was prepared in relation to the wireless power transmission to the ECG sensor. To establish a wireless network-driven environment for transmitting and receiving ECG data for measurement, a device was prepared that emitted an electric current identical to that of a real ECG and connected to the ECG sensor. An infrared temperature camera was then used to measure the temperature changes of the sensor itself; temperature changes were measured prior to the initiation of power transmission and then continued for one hour after transmission began with the aim of determining the average temperature change. Figure 4 shows the thermal testing experimental setup.

From the experiments, we found that the baseline average temperature was $23.5^{\circ} \mathrm{C}$. The temperature then sharply rose by about $3.0^{\circ} \mathrm{C}$ after about 10 minutes and continued to rise to reach $27.2^{\circ} \mathrm{C}$ after one hour. As this temperature is far below $36.9^{\circ} \mathrm{C}$-the average temperature of the interior of the human body-it can be assumed that the packaged sensor will not undergo considerable temperature change once inserted into the human body. Figure 5 shows the thermal testing experimental setup and Figure 6 shows the insertion experiment using animal model.

3.3. Insertion Experiment Using Animal Model. Before using the sensor within a human body, it was necessary to test the in vivo safety of the instrument to ensure both the efficient operation of the insertion-type ECG measurement system in
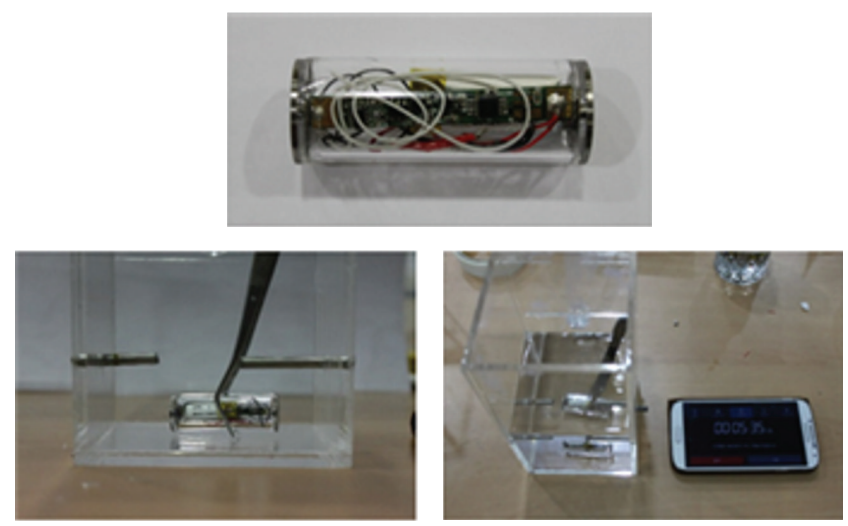

FIGURE 4: Self-sealing test using the packaged sensor.

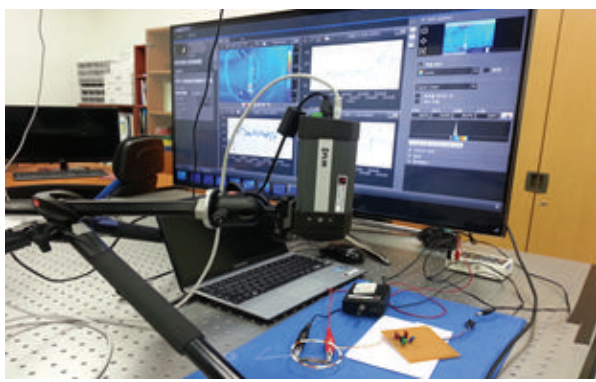

FIGURE 5: Thermal testing experimental setup.

measuring physiological functions and its efficacy in receiving external signals. A pig was therefore used as a sensorimplanted animal model because the animal's physiological characteristics are similar to those of humans. The species selected was a Hanford mini pig because its heart size is very similar to that of a human. A female pig of specific pathogen free (SPF) quality with no history of pregnancy, 46-60 kg, and 50-57-week old, was purchased from Optipharm Medipig (Chungbuk, South Korea).

The insertion surgery was performed in the Daegu HighTech Medical Complex as follows. Anesthesia was induced using Zoletil (Tiletamine/Zolazepam) $(2.5 \mathrm{mg} / \mathrm{kg}$, IM) and Xylazine $(2.3 \mathrm{mg} / \mathrm{kg}, \mathrm{IM})$ and maintained with Isoflurane (1$3 \%)$. Lactated ringer's solution $(5 \mathrm{~mL} / \mathrm{kg} / \mathrm{h}, \mathrm{IV})$ was administered intraoperatively. After the anesthesia, the left anterior corselet was depilated and disinfected with alcohol and povidone. An incision was made between the left 5 th and 7 th ribs and separated using blunt dissection to a depth of $4 \mathrm{~mm}$ under the skin. The sensor was placed at the site, and the skin was sutured. On completion of the wireless ECG sensor implantation, the pig's ECG data were received by wireless network, as shown in Figure 7. In this data, two leads were continuously corrected. In the current experiments, we tried a short-term animal experiment. In the future work, the longterm experiment will be conducted to guaranty the proposed sensor safety issue.

\section{Discussions}

The current power consumption is relatively high due to the real-time communication between implanted sensor and 

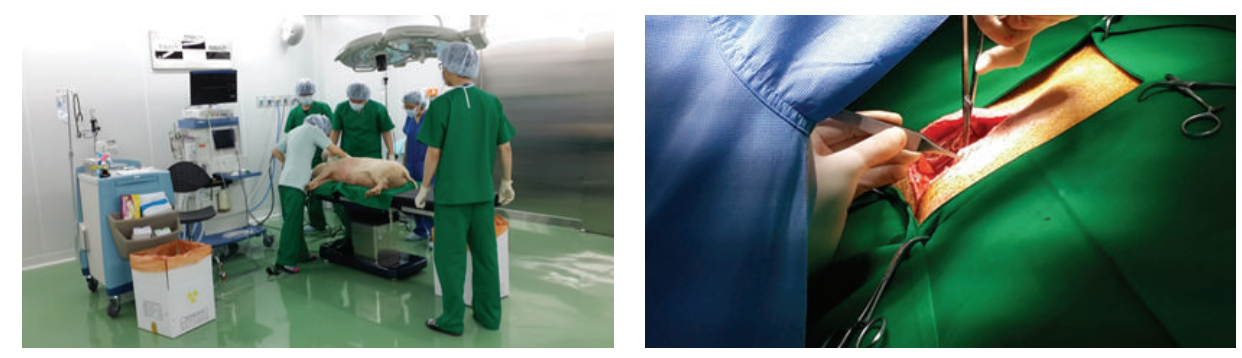

FIGURE 6: Insertion experiment using animal model.

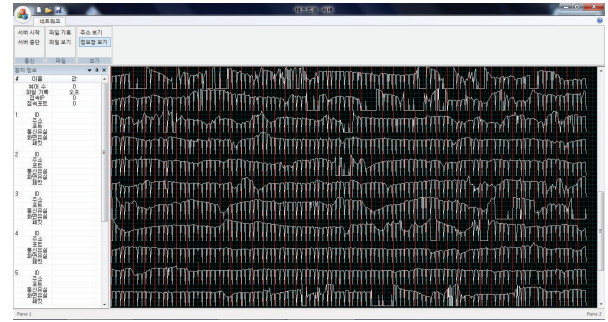

FIGURE 7: ECG monitoring results using animal model.

outside workstation with $300 / \mathrm{sec}$ sampling rate. To decrease the power consumption, the lower sampling rate or the discrete communication method can be considered in the future work. In the current animal experiments, two pigs were studied. The pig with similar human weights was considered with the IRB (institutional review board) guideline. In this paper, we suggested the real-time data transmission device. The reason is that the raw data should be corrected to determine the abnormality of ECG from doctors without computer aided diagnosis system.

\section{Conclusion}

This study demonstrated that the use of a quasipermanent ECG employing a double loop coil-shaped magnetic resonance-type wireless power transmission system sensor eliminates the need for surgical replacement. An ultrasmall antenna (20 $\mathrm{mm}$ in width, $10 \mathrm{~mm}$ in length) with a spiralshape metal pattern was developed and used to minimize sensor size while securing a sufficient electric length. A human body phantom that had similar electrical properties to those of human skin within the MICS band, with a $10 \%$ error range of measurement values (specific permittivity $=$ 43.2 , conductivity $=0701 \mathrm{~S} / \mathrm{m}$ ), was developed and used to verify the communication performance of the antenna. The hermetic joining of packaging using adhesives and biocompatibility were also experimentally verified. Finally, sensor insertion surgery was performed on a laboratory pig and successful ECG data were obtained via a wireless network.

\section{Conflict of Interests}

The author declares that there is no conflict of interests regarding the publication of this paper.

\section{Acknowledgments}

This work was supported by a Grant (R0101-15-0147) from the Technology Innovation Program funded by the Ministry of Knowledge Economy (MKE, Republic of Korea) and supported by the R\&D Program of the Ministry of Trade, Industry and Energy of Korea (Program of Advanced Technology Development for Future Industry, 10044353), and supported by Basic Science Research Program through the National Research Foundation of Korea (NRF) funded by the Ministry of Education (2014R1A1A2056420).

\section{References}

[1] Y. Hao and R. Foster, "Wireless body sensor networks for health-monitoring applications," Physiological Measurement, vol. 29, no. 11, pp. R27-R56, 2008.

[2] J. D. Newman and A. P. F. Turner, "Home blood glucose biosensors: a commercial perspective," Biosensors and Bioelectronics, vol. 20, no. 12, pp. 2435-2453, 2005.

[3] IEEE Standard 1528/D1.2, "Recommended Practice for determining the spatial-peak specific ab-sorption rate (SAR) in the human body due to wireless communications devices: measurement techniques," 2003.

[4] A. Pantelopoulos and N. G. Bourbakis, "A survey on wearable sensor-based systems for health monitoring and prognosis," IEEE Transactions on Systems, Man and Cybernetics, Part C: Applications and Reviews, vol. 40, no. 1, pp. 1-12, 2010.

[5] J. Malmivuo and R. Plonsey, Bioelectromagnetism, Oxford University Press, New York, NY, USA, 1995.

[6] P. J. Zimetbaum and M. E. Josephson, "The evolving role of ambulatory arrhythmia monitoring in general clinical practice," Annals of Internal Medicine, vol. 130, no. 10, pp. 848-856, 1999.

[7] G. E. Bergey, R. D. Squires, and W. C. Sipple, "Electrocardiogram recording with pasteless electrodes," IEEE Transactions on Biomedical Engineering, vol. BME-18, no. 3, pp. 206-211, 1971.

[8] A. Searle and L. Kirkup, "A direct comparison of wet, dry and insulating bioelectric recording electrodes," Physiological Measurement, vol. 21, no. 2, pp. 271-283, 2000.

[9] G. Cho, K. Jeong, M. J. Paik, Y. Kwun, and M. Sung, "Performance evaluation of textile-based electrodes and motion sensors for smart clothing," IEEE Sensors Journal, vol. 11, no. 12, pp. 3183-3193, 2011.

[10] J. Mühlsteff and O. Such, "Dry electrodes for monitoring of vital signs in functional textiles," in Proceedings of the 26th Annual International Conference of the IEEE Engineering in Medicine and Biology Society (EMBC '04), pp. 2212-2215, San Francisco, Calif, USA, September 2004. 
[11] R. Matthews, N. J. McDonald, I. Fridman, P. Hervieux, and T. Nielsen, "The invisible electrode zero prep time, ultra low capacitive sensing," in Proceedings of the 11th International Conference on Human-Computer Interaction, pp. 22-27, Las Vegas, Nev, USA, 2005.

[12] Y. M. Chi and G. Cauwenberghs, "Wireless non-contact EEG/ECG electrodes for body sensor networks," in Proceedings of the International Conference on Body Sensor Networks (BSN '10), pp. 297-301, IEEE, Singapore, June 2010.

[13] A. D. Droitcour, O. Boric-Lubecke, V. M. Lubecke, J. Lin, and G. T. A. Kovacs, "Range correlation and I/Q performance benefits in single-chip silicon Doppler radars for noncontact cardiopulmonary monitoring," IEEE Transactions on Microwave Theory and Techniques, vol. 52, no. 3, pp. 838-848, 2004. 


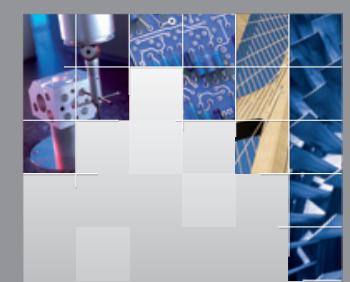

\section{Enfincering}
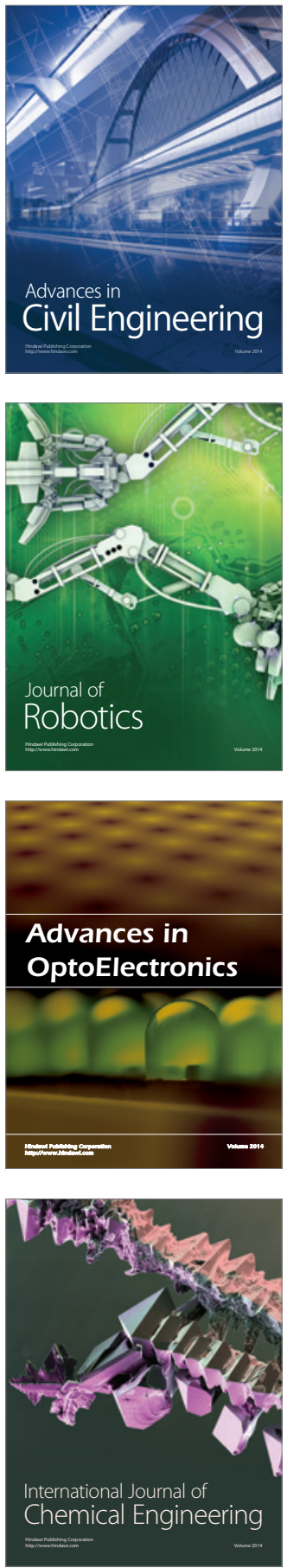

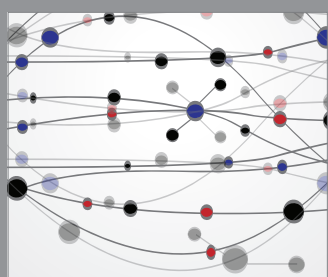

The Scientific World Journal

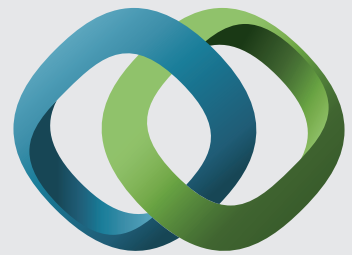

\section{Hindawi}

Submit your manuscripts at

http://www.hindawi.com
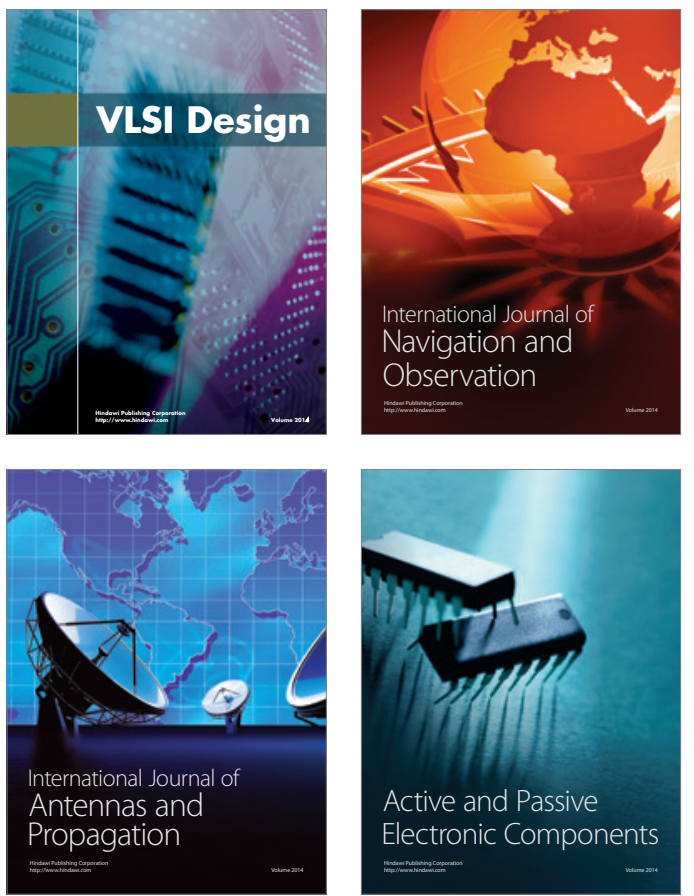
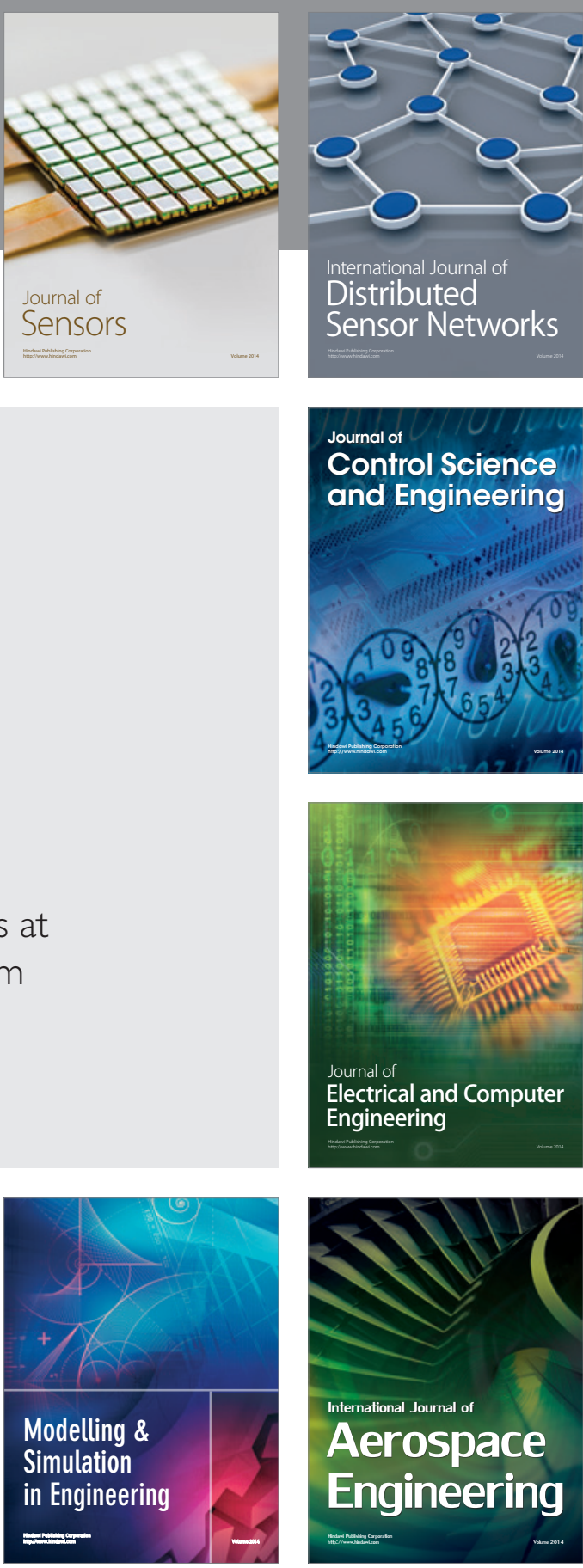

International Journal of

Distributed

Sensor Networks

Journal of

Control Science

and Engineering
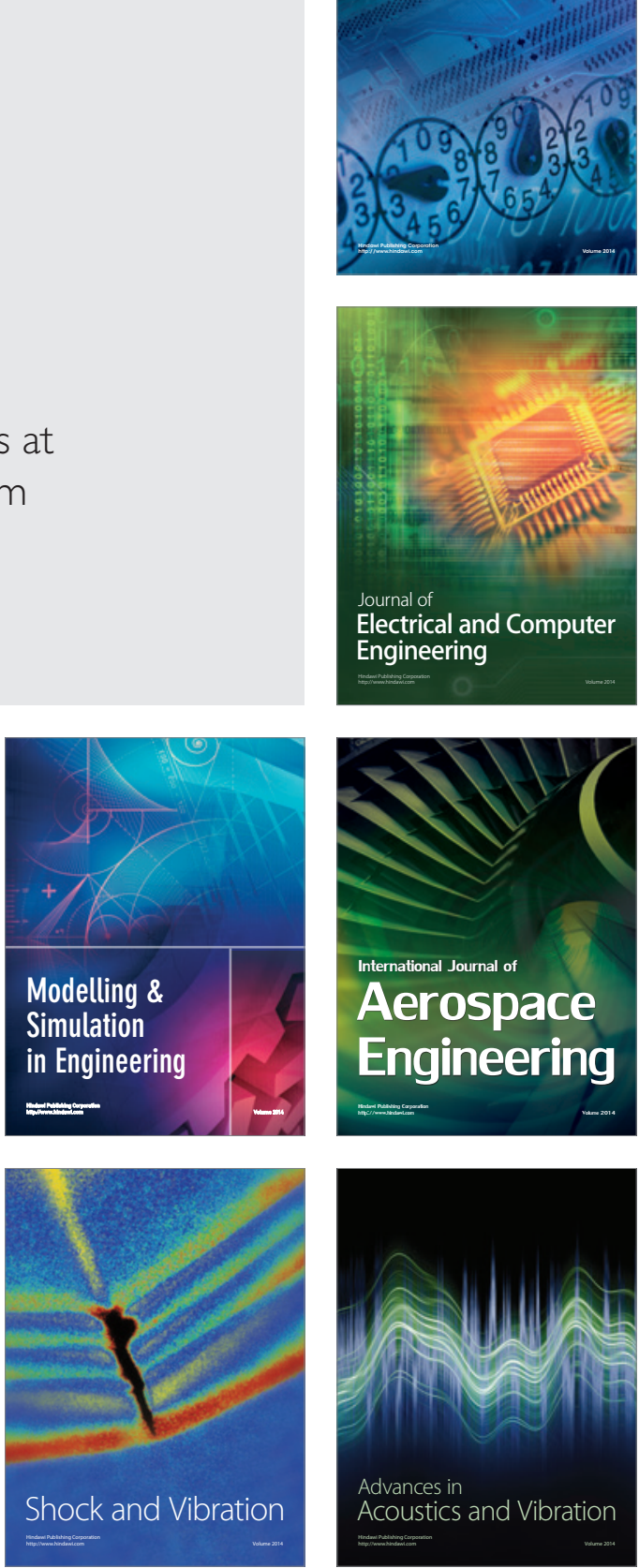